\title{
5GENESIS: The Genesis of a flexible 5G Facility
}

Harilaos Koumaras ${ }^{1}$, Dimitris Tsolkas ${ }^{2}$, Georgios Gardikis ${ }^{3}$, Pedro Merino Gomez ${ }^{4}$, Valerio Frascolla ${ }^{5}$, Dionysia Triantafyllopoulou ${ }^{6}$, Marc Emmelmann ${ }^{7}$, Vaios Koumaras ${ }^{8}$, Maria L. Garcia Osma ${ }^{9}$, Daniele Munaretto ${ }^{10}$, Eneko Atxutegi ${ }^{11}$, Jara Suárez de Puga ${ }^{12}$, Ozgu Alay ${ }^{13}$ Anna Brunstrom ${ }^{14}$, Anne Marie Cristina Bosneag ${ }^{15}$

${ }^{1}$ National Centre for Scientific Research "DEMOKRITOS”, Greece, ${ }^{2}$ Fogus Innovations \& Services, Greece, ${ }^{3}$ Space Hellas

(Cyprus) Ltd., Greece, ${ }^{4}$ Universidad de Málaga, Spain, ${ }^{5}$ Intel Deutschland GmbH, Neubiberg, Germany, ${ }^{6}$ University of Surrey (5G Innovation Centre - 5GIC), UK, ${ }^{7}$ FOKUS-Fraunhofer Gesellschaeft e.V., Germany, ${ }^{8}$ INFOLYSiS P.C., Greece, ${ }^{9}$ Telefónica, Spain, ${ }^{10}$ Athonet, Italy, ${ }^{11}$ Nemergent Solutions SL, Spain, ${ }^{12}$ Universitat Politècnica de València, Spain, ${ }^{13}$ Simula Research Laboratory, Norway, ${ }^{14}$ Karlstad University, Sweden, ${ }^{15}$ Ericsson LMI, Ireland.

\begin{abstract}
This paper describes the pathway towards the realisation of a $5 \mathrm{G}$ Facility that will allow the validation of the major 5G Key Performance Indicators (KPIs). It reflects the approach that the 5GENESIS consortium will adopt in this direction. More precisely, it describes the key design principles of such Facility as well as the targeted use cases for the KPIs validation. The adopted approach for the Facility realisation includes the design of a common implementation blueprint that will be instantiated in five Platforms distributed across Europe. To maximise the diversity and the efficiency of the Facility, complementary performance objectives have been selected for the Platforms, while specific characteristics from different vertical industries have been allocated to each of them.
\end{abstract}

Keywords - 5G Facility; Testbeds; Experimentation; Key Performance Indicators; Virtualization; Slicing.

\section{INTRODUCTION}

The fifth generation of mobile technology (5G) is positioned to address the demands and business contexts of IMT 2020 and beyond [1]. Standards Development Organizations (SDOs), industry fora and research projects have identified as common ground the need for: i) enhancing the network architecture, in view of emerging technologies and features, and ii) pushing the envelope of performance, both from network and user perspectives, towards ultimate capabilities far beyond $4 \mathrm{G}$, in support of a wide range of innovative use cases [2] to suit various verticals. In this direction, several international research projects, [3], [4], address the challenge of proposing $5 \mathrm{G}$ testbeds. Especially under the EU-funded FP8 umbrella, the so-called 5G-PPP Phase 1 projects mapped the $5 \mathrm{G}$ requirements to specific Key Performance Indicators (KPIs) and formulated the overall $5 \mathrm{G}$ architecture that will achieve these KPIs. The ongoing 5GPPP Phase 2 projects are extending the performed work in Phase 1 , by contributing to a selective experimentation of different technologies needed to validate the $5 \mathrm{G}$ architecture. With significant contributions from the research work of the $5 \mathrm{G}-\mathrm{PPP}$ projects, the $3 \mathrm{GPP}$ standardization body is working on the $5 \mathrm{G}$ standard incorporating end-to-end (E2E) advances for the mobile network. Finally, the $5 \mathrm{G}$ Infrastructure Association (5G-IA) has already defined the roadmap towards a 5G Pan-European trial network [5].

Irrefutably, the "Genesis of $5 \mathrm{G}$ " has entered the crucial phase of experimentation, and currently faces the challenge of validating the $5 \mathrm{G}$ network KPIs and verifying the $5 \mathrm{G}$ technologies with an E2E approach. Towards this objective, a key challenge is to integrate all the highly diverse results and technologies from R\&D projects, to "glue together" the $5 \mathrm{G}$ picture and unveil the potential of a truly full-stack and E2E $5 \mathrm{G}$ Facility, to meet the targeted KPIs.

In this context, the paper describes the key design principles and the innovative 5G Facility that the recently EUfunded 5GENESIS [6] research project will build. The novel Facility will be composed of several so-called Platforms, i.e. enhanced existing $5 \mathrm{G}$ testbeds and related applications, which aim to address some societal challenges and still not fully solved technical aspects of $5 \mathrm{G}$ networks. Each Platform forms a validation setup and the combination of all Platforms build an open, flexible and distributed experimentation facility.

The remainder of the paper is organized as follows. Section II provides the main design principles of the $5 \mathrm{G}$ Facility, Section III describes its components, and Section IV the reference implementation blueprint. The use cases targeted for demonstration are depicted in Section V. The conclusions of the paper are given in section VI.

\section{THE DESIGN PRINCIPLES}

The focus of our work is to establish a complete, open, evolving and distributed experimentation facility across Europe, towards assessing the added-value of $5 \mathrm{G}$ and validating the associated KPIs. Taking into account the 5GPPP recommendations as well as the current 5G landscape, 5GENESIS identifies the following design principles and key requirements, upon which it builds its concept.

- The 5GENESIS Facility shall be geographically distributed across Europe and comprise various Platforms. Instead of building a centralized infrastructure, 5GENESIS leverages existing assets and testbeds across Europe and evolves them to include 5G technologies, thus establishing a fully distributed experimentation facility. In this manner, technological developments are more focused and less costly, exploiting already deployed local assets and human resources. Furthermore, the access to the Facility is significantly improved, since experimenters can choose the Platform which most suits their needs, in terms of technological capabilities and geographical proximity.

- The Platforms shall be complementary in terms of features, while still aligned to a common reference architecture (experimentation blueprint, as described in section IV). For the sake of resource and cost efficiency, each Platform focuses on a specific set of technological associated KPIs. These features and KPIs are mapped to the platforms in a complementary manner so that, as a 
whole, the 5GENESIS Facility shall be able to address all $5 \mathrm{G}$ PPP KPIs.

- The Platforms shall be administratively independent, exposing open interfaces for inter-platform coordination, integration and verticals experimentation. A top-level management (federation) entity for the whole Facility is not foreseen. Instead, each Platform is independently managed and horizontally interconnected with the other platforms for inter-domain services. This approach promotes resilience and scalability and greatly facilitates platform management, being directly analogous to the operational ISP and Telco infrastructures.

- The Platforms shall accommodate multiple experiments from various verticals and shall be able to simultaneously accommodate multiple services with different requirements (e.g., eMBB, uRLLC and mMTC) on the same infrastructure, hence, fulfilling one of the key features of $5 \mathrm{G}$. This capability will exist in all platforms, driven by network virtualization and E2E slicing.

- Each Platform will expose common APIs for interacting with experimenters, thus promoting openness, facilitating trials from vertical industries across Europe and enabling portability of experiments on the Platforms.

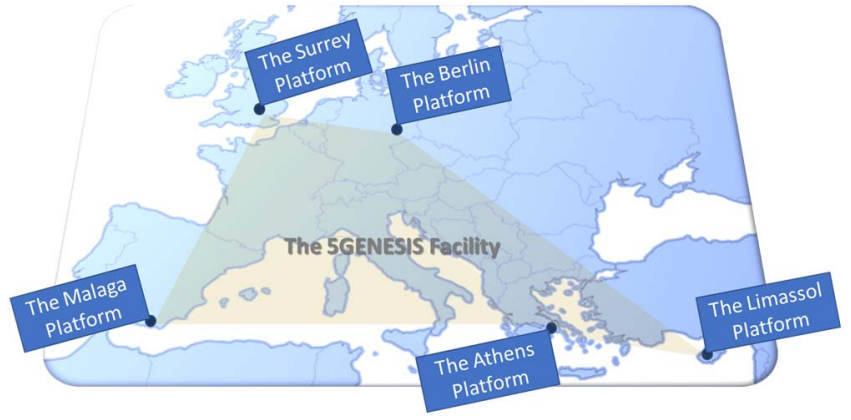

Figure 1: Distribution of the Platforms around Europe

\section{THE INGREDIENTS OF THE 5GENESIS FACILITY}

\section{A. Brief overview}

The five platforms of the 5GENESIS Facility (Figure 1) and their main features are as follows:

- The Athens Platform provides a shared radio infrastructure (gNBs and small cells) with different ranges and overlapping coverage that is supported by an SDN/NFV enabled core and enabled edge-computing capabilities. It is conceived to showcase scenarios where multimedia content delivery and low latency applications coexist in large public-events.

- The Málaga Platform brings automated orchestration and management of different network slices over multiple domains, on top of the $5 \mathrm{G}$ New Radio (NR) access and fully virtualised core network to showcase Mission Critical Services (MCS) running in an Edge Computing platform and outdoor deployments.
- The Limassol Platform offers radio interfaces of different characteristics and capabilities, combining terrestrial and satellite communications, integrated to showcase service continuity and ubiquitous access in underserved areas together with interoperability of IoT components deployed within these areas.

- The Surrey Platform features multiple radio access technologies that can support massive Machine Type Communications (mMTC), including 5G NR and NBIoT, combined under a flexible Radio Resource Management (RRM) and spectrum sharing platform to showcase massive IoT services.

- The Berlin Platform contains ultra-dense areas covered by various network deployments, ranging from indoor nodes to nomadic outdoor clusters, coordinated via advanced backhauling technologies to showcase immersive service provisioning.

\section{B. Alignment with the 5G-PPP KPIs}

In alignment with the 5G-PPP targets for the experimentation phase of 5G, the 5GENESIS Facility will mainly be used to validate the following performance indicators:

\section{a) Service level Performance Indicators}

Data rates. Athens, Limassol, Berlin and Málaga platforms will demonstrate high data rates for eMBB. For example, Málaga will demonstrate the concurrent use of 10 to 20 cameras with real time high quality video in a small area (300m x 300m), with several configurations combining LTE, $5 \mathrm{G} \mathrm{NR}$ and Optical fibre to balance the traffic distribution.

Reliability and latency. Málaga Platform will demonstrate Mission Critical Services (MCPTT, MCVideo and MCData, based on 3GPP R14) in both lab and outdoor deployments. Although the current KPIs defined by 3 GPP in release 13 are not so strict to URLLC applications (e.g. tele-surgery), the same deployments will be used to demonstrate latency $<10 \mathrm{~ms}$, reliability $>99 \%$ and availability $>99 \%$. Experiments conducted indoor in laboratories could demonstrate even more ambitious performance KPIs.

\section{b) Application level Performance Indicators}

The 5GENESIS Facility will be instrumental in also assessing the overall service improvement, by measuring application-level indicators, such as: i) the user-perceived quality of experience (QoE), ii) the impact to the E2E security guarantees.

Quality of Experience. The QoE assessment will be studied in the Athens, Málaga and Berlin Platforms, where Human Type Communications (HTC) are foreseen. The validation concept will involve real end-users in the assessment process combined with objective QoE estimation methods [9]. The Berlin Platform will evaluate the provided network quality over a heterogeneous set of backhauling nodes, using mmWave and optical fibre links. The Málaga Platform will 
evaluate QoE with the Police Department in the context of Mission Critical Services.

Security. Regarding the security-related assessment, the Athens platform will be responsible for examining the security in a dense urban environment for demanding video services. The goal here is to provide a content delivery framework by taking advantage of the SDN/NFV technologies without affecting security or violating privacy.

\section{c) Network Level Performance Indicators}

Coverage. The Limassol platform will demonstrate ubiquitous $5 \mathrm{G}$ coverage, with scenarios oriented to maritime communications, rural and underserved areas. The platform will combine satellite communications links with terrestrial backhaul and access technologies, employing advanced link aggregation and optimisation technologies, integrating them all in the overall $5 \mathrm{G}$ network.

Density. The Surrey platform will support mMTC in a network of densely distributed NB-IoT and LoRa nodes. In the demonstration event, the platform will be stressed to achieve 1000X simultaneously connected devices, starting with $\sim 1000$ real nodes and pushing the system to the limits with additional set of simulated ones.

Network lifecycle management. Performance metrics such as the slice establishment time, the VNF relocation and instantiation times, and the computational resource usage of the cloudified protocol stack, will also be considered. Such metrics will be quantified in the Surrey and Málaga platforms. In this regard, the goal is to exemplify in an E2E fashion the trade-off between the flexibility required in the $5 \mathrm{G}$ networking and the constraints and limitations of the virtualization and ETSI MANO frameworks to be used. These measurements will also provide a way to understand the type and benefits of self-adaptable mechanisms that can be put in place to minimise the gap between the flexibility and constraints provided by virtualisation.

Table I summarizes the distribution of the major targeted KPIs among the Platforms of the 5GENESIS Facility.

Table I: Allocation of major network KPIs to the $5 \mathrm{G}$ platforms

\begin{tabular}{|c|c|c|c|c|c|}
\hline KPI Platform & Málaga & Athens & Limassol & Surrey & Berlin \\
\hline Capacity & $\checkmark$ & $\checkmark$ & & & \\
\hline Ubiquity & & & $\checkmark$ & $\checkmark$ & \\
\hline Speed & $\checkmark$ & $\checkmark$ & & $\checkmark$ & $\checkmark$ \\
\hline Latency & $\checkmark$ & $\checkmark$ & $\checkmark$ & $\checkmark$ & \\
\hline Reliability & $\checkmark$ & & $\checkmark$ & $\checkmark$ & $\checkmark$ \\
\hline Density of Users & $\checkmark$ & & & $\checkmark$ & $\checkmark$ \\
\hline Location accuracy & $\checkmark$ & & & & \\
\hline Energy efficiency & & & & $\checkmark$ & \\
\hline Service creation time & $\checkmark$ & $\checkmark$ & $\checkmark$ & & $\checkmark$ \\
\hline $\begin{array}{c}\text { Network management } \\
\text { CAPEX/OPEX }\end{array}$ & $\checkmark$ & $\checkmark$ & & & \\
\hline
\end{tabular}

\section{REFERENCE IMPLEMENTATION BLUEPRINT}

The proposed reference architecture inherits several concepts from relevant $3 \mathrm{GPP}$ documents such as TR 28.801, TR
32.864, TR 23.714, TS 23.214, and TR 23.799 as well as from the ETSI NFV ISG (GS NFV 002, GS NFV MAN 001, etc.) and ETSI GS MEC (GS MEC 003) initiatives. It is also compliant with the $5 \mathrm{G}$ generic architecture, as defined by 5G-PPP [7]. As seen in Figure 2, the reference implementation blueprint consists of three main layers:

- Coordination - A Platform Coordinator component is responsible for the overall coordination of the Facility, achieving overall supervision and E2E configuration for service deployment, management and monitoring.

- Management and Orchestration - It includes: i) the slice management module; ii) MANO components for the NFV and MEC infrastructures of the Facility, iii) SDN controllers for the programmable network as well as iv) traditional Network Management System functionalities for controlling the Physical Network Functions (PNFs), mostly involving the radio and $5 \mathrm{G}$ core components.

- Infrastructure - It involves the E2E components which handle the user traffic, including the virtualised core network functionalities running on an NFVI (Network Functions Virtualization Infrastructure), the backhaul links, the mobile edge node and the radio heads, as well as the end-user equipment. Both the $5 \mathrm{G}$ Core Network Functions (split into control and user plane VNFs) as well as the Access Network Functions (VSFs, components of the spectrum management framework) and the user VNFs, are virtualised and distributed, according to their nature and functionalities, across the core and edge virtualisation infrastructures, thus enabling the Control and User Plane Separation (CUPS).

It must be emphasized that the proposed reference architecture is fully modular according to the $5 \mathrm{G}$ architecture requirements in $3 \mathrm{GPP}$, allowing different configurations to be instantiated in each of the different 5GENESIS Platforms, and eventually enabling different capabilities of $5 \mathrm{G}$ to be exploited in each case.

\section{TARgeted USE CASES}

The use cases have been carefully selected to cover a wide range of complementary $5 \mathrm{G}$ showcasing scenarios.

The Athens Platform will demonstrate how 5G enables innovative applications for drastically augmenting the creation of content and the experience of the audience in big events (sports, cultural, etc.). The Egaleo municipal stadium will be the place where these events will take place. More precisely, two service scenarios are foreseen:

- Content creation: this scenario will demonstrate adaptive upstream content transmission via the $5 \mathrm{G}$ network. It will assume a low-cost content producer with inexpensive cameras covering the event. Without the need for employing costly microwave links and satellite connections for video streaming transmission and also avoiding the overhead on the cellular macro cell infrastructure (Figure 3), the content producer employs 


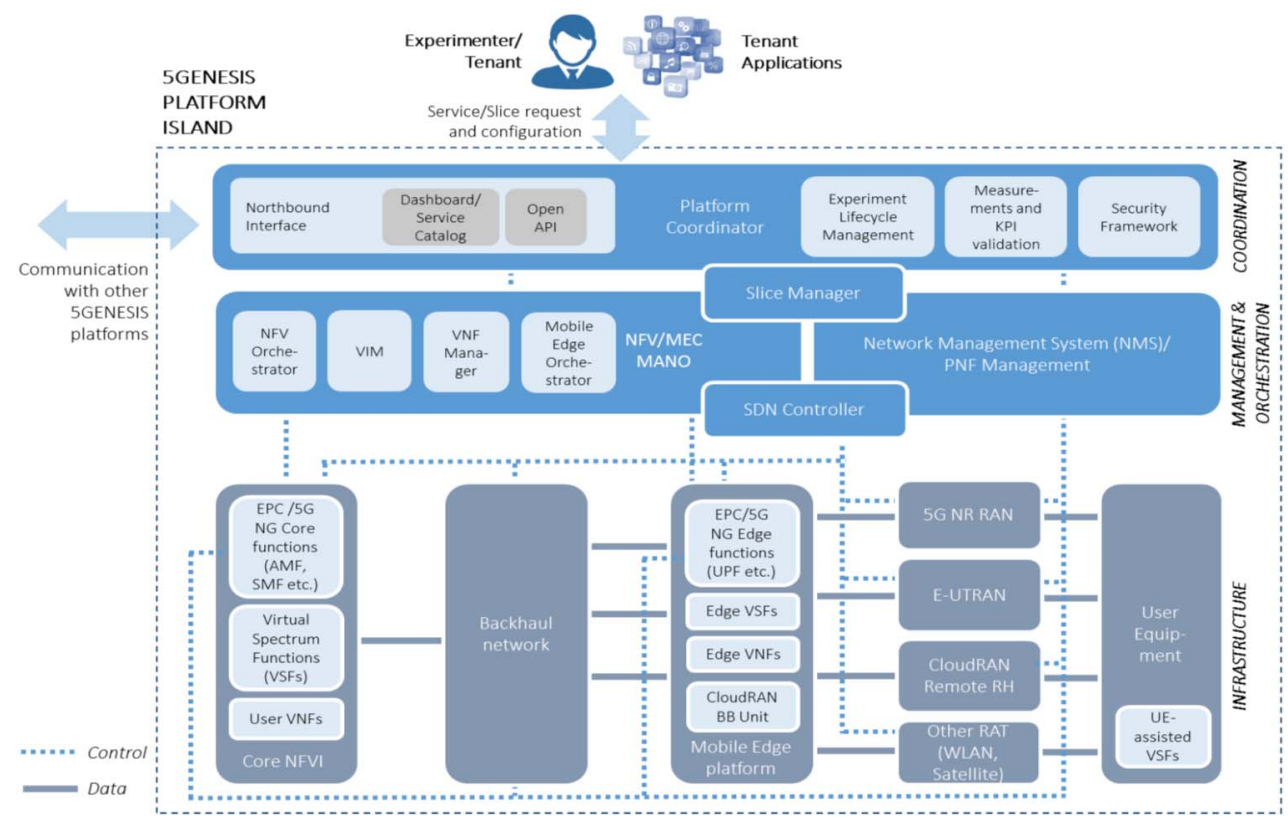

Figure 2: Reference Implementation Blueprint

a service slice over the stadium's cloud-enabled small cell (CESC) infrastructure with both guaranteed QoE and network edge processing (adaptive multimedia transcoding) and connectivity (MEC), to allow local traffic to stay local and thus meeting the high-throughput and low latency requirements.

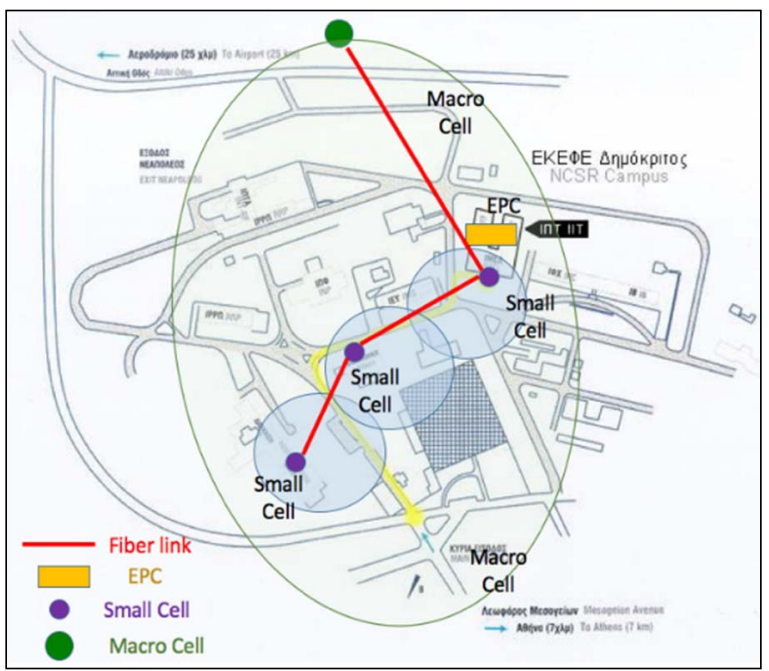

Figure 3: The 5G Infrastructure deployed at the National Centre for Scientific Research "Demokritos", to be exploited by the 5GENESIS Facility.

- Low-latency AR (Augmented Reality) applications: the second scenario will be based on an AR application, to be developed in the project and installed in the smartphones of the audience. Using the AR application and the smartphone camera, the users can focus on any object in the scene (e.g., a particular player in a football match) and instantly receive either real-time information about the object or watch a video stream (served by the content producer, see previous scenario). The mobile edge computing infrastructure will be used to i) host part of the AR application, thus reducing the processing overhead at the mobile device, and ii) serve the associated content, thus relieving the backhaul link and drastically reducing the response time (low latency is critical for AR applications).

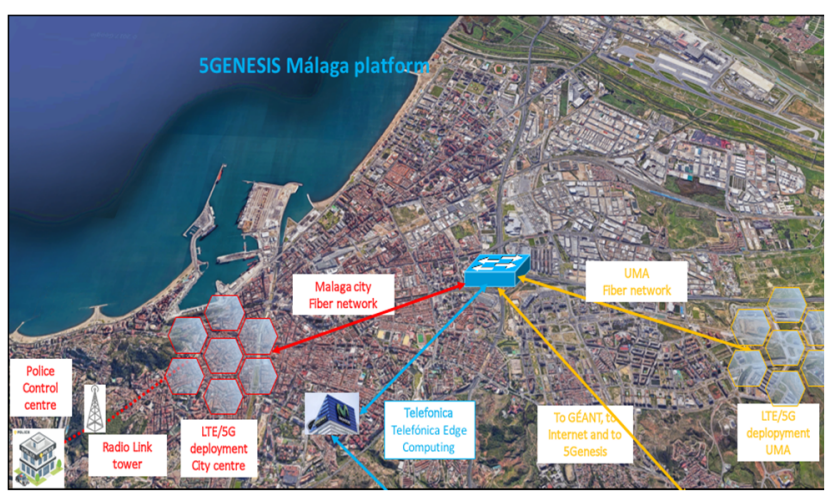

Figure 4: The Malaga platform components to be exploited by the 5GENESIS Facility

The Málaga Platform will test MCS in the city of Málaga using the deployment depicted in Figure 4. Since current Professional Multi Radio (PMR) services are still limited to narrowband voice when using technologies such as TETRA and TETRAPOL, The Málaga Platform will make possible the deployment of MCS for several configurations over 3 GPP networks. MCSs will request specific capabilities to the network with standard 3GPP interfaces and APIs that the platform will make available to third-party applications and legacy systems. Several terminals are considered, including cameras deployed on the fly and specific equipment to be carried by Police agents. The measurement of all the relevant $5 \mathrm{G}$ KPIs for this use case will be included in the context of 
the QoE evaluated by real users. How custom terminals using MCS can operate E2E will also be demonstrated. For instance, slices could be established for Video, Data, Voice (3GPP MCPTT R13 services) and eMBMS support.

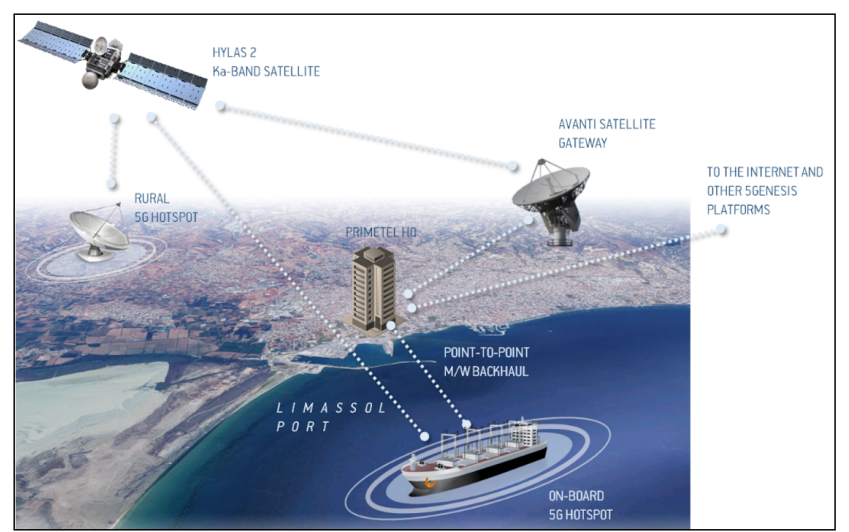

Figure 5: Satellite-supported infrastructure at the Limassol port, to be exploited by the 5GENESIS Facility

The Limassol Platform will be used to demonstrate two scenarios, one related to $5 \mathrm{G}$ maritime communications and the other related to $5 \mathrm{G}$ services in rural/underserved areas including the management and interconnection of heterogeneous IoT devices and platforms existing in $5 \mathrm{G}$ environments (Figure 5).

- Seamless Maritime Communications: Validation of the "5G hotspot" on the vessel (tanker), served by a hybrid satellite/terrestrial backhaul and evaluating local and remote real-time multimedia communication, as well as sensor network interconnection. Candidate users: Shipping companies (passenger and cargo ships), yacht owners, oil rig holders, and so on.

- $5 G$ capacity-on-demand in rural/underserved areas: Adhoc deployment of a "5G hotspot" and IoT access gateways within areas not (adequately) covered by the existing cellular network infrastructure. Scenarios will include capacity boost for a flash crowd event, administration and interoperability of IoT sensor systems connected to the network and/or the dynamic provision of network slices for multimedia services for large-scale events in rural/underserved areas. Candidate users: Event organisers, local authorities, first responders and public safety, hotel owners, TV and media broadcasters (nextgeneration Satellite News Gathering). Applications will focus on voice/video communication as well as sensor network interconnection, using the IoT interoperability service (see Sec. III. Both use cases, especially the first one, are of particular relevance to the Limassol stakeholder ecosystem, in which the maritime sector is key element. The evaluation plan also includes demonstration and assessment in open public events, such as the Mediterranean Science Festival in Limassol.

The Surrey Platform will be used for the demonstration of effective massive IoT and multimedia communications in a
multi-RAT and multi-spectrum licensing scheme environment, by employing novel RRM solutions. More specifically, a 5G NR and a number of RATs, including LTEA, WiFi, NB-IoT and LoRa, will be deployed in the area of the Surrey Sports Park (SSP), one of Europe's premier sites for sport and leisure (Figure 6). The aim is to validate $5 \mathrm{G}$ KPIs during large scale sports events, mainly benefitting the eHealth and, the multimedia and entertainment verticals. Specifically, the particular use case that is targeted aims at providing an immersive experience with pervasive connectivity during ad-hoc and large scale come-togethers (sport events, concerts, special events). To this end, a massive IoT network slice combined with a dynamic eMBB slice will facilitate the use of information provided by the audience terminals and by a variety of sensors deployed in the venue, for the provision of up to date information and improvement of the provided in-stadium services. The flexible RRM will be supported by self-adaptive policy mechanisms, and intelligent analytics and learning mechanisms. Moreover, the use case considers the real-time monitoring of athletes' healthcare information during a sports event. The rich, truly connected user experience will be complemented by the use of an eMBB slice that will allow the uploading, sharing and curation of real-time video generated by the audience.

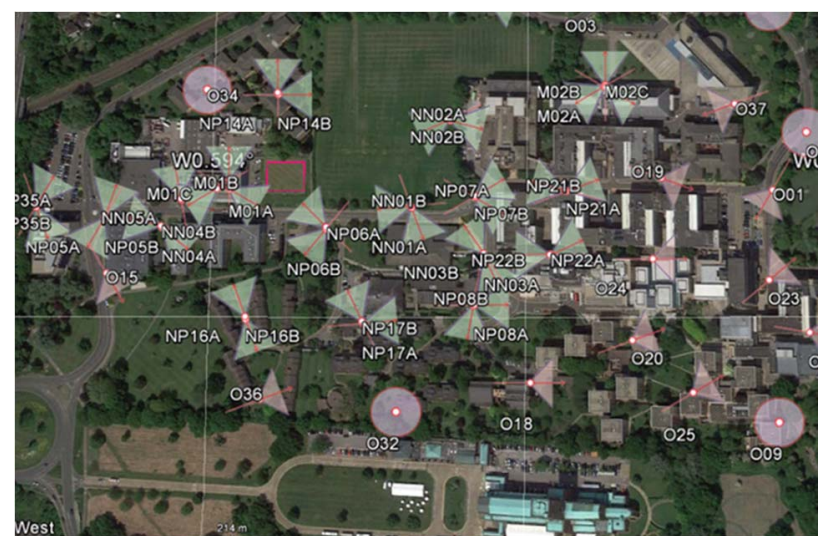

Figure 6: Outdoor deployment of IoT AP to be exploited by the 5GENESIS Facility

The Berlin Platform will assess a "Dense Urban Use Case" realizing 360deg VR streaming. The platform will be used to provide network connectivity at the Humboldt-University during the "Festival of Lights Berlin", which is attended by thousands of visitors (Figure 7). The "dense urban use case" will demonstrate to future European R\&D projects, industry, and SMEs that the Berlin platform can be used for $5 \mathrm{G}$ evaluations involving a myriad of public users. The chosen 360deg VR application will be of interest to the city of Berlin and local (media) businesses, hence will likely increase the visibility of the trial. Additionally, the targeted use case will allow the validation of $5 \mathrm{G}$ PPP KPIs in a real urban environment. It is expected that the trial on the Berlin platform will provide insights on how $5 \mathrm{G}$ advances the stateof-the-art regarding the following use of: i) Mobile Edge Computing [8] to reduce latency and achieve high 
throughput, ii) VNFs to support slicing, iii) cloud utilities to reduce service creation time, and iv) link aggregation across multiple RATs to increase reliability and resilience.

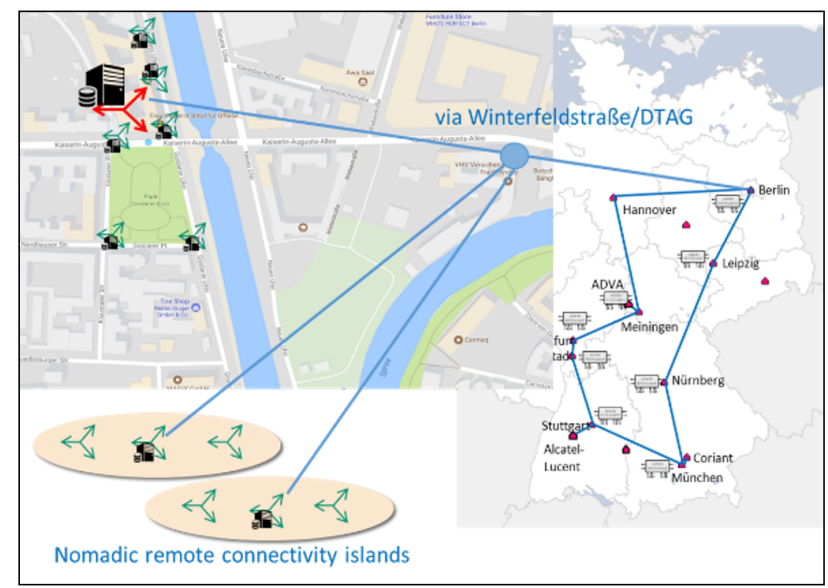

Figure 7: 5G Berlin outdoor deployment, with nomadic remote connectivity islands, to be exploited by the 5GENESIS Facility

\section{CONCLUSIONS}

This paper introduces an innovative approach for the development and evaluation of a flexible 5G E2E network facility. The 5GENESIS Facility can be used for experiments verifying $5 \mathrm{G}$ KPIs, as well as for additional demonstrations and evaluations of some key $5 \mathrm{G}$ and beyond use cases. The trials will focus on verifying key 5G-PPP KPIs and hence implicitly assessing the capabilities and performance of each platform that composes the 5GENESIS Facility. In summary, the 5GENESIS will facilitate the move beyond the current state of play in $5 \mathrm{G}$ experimentation with a novel design of a 5G-capable experimentation facility and its instantiation for showcasing in five different use cases. This step forward in the network design is reflected in the proposed reference implementation blueprint.

\section{ACKNOWLEDGEMENTS}

This work has been conducted under the auspices of the 5GENESIS project. This project has received funding from the European Union's Horizon 2020 research and innovation programme under grant agreement No 815178 .

\section{References}

[1] http://www.itu.int/en/ITU-R/study-groups/rsg5/rwp5d/imt2020/Pages/default.aspx

[2] https://www.ngmn.org/fileadmin/ngmn/content/downloads/T echnical/2015/NGMN_5G_White_Paper_V1_0.pdf

[3] P. Marques, et al., "Experiments Overview of the EU-Brazil FUTEBOL Project" in 26th European Conference on Networks and Communications (EuCNC), 2017.

[4] M. Mueck, et al., "5G CHAMPION - Rolling out 5G at 2018 Winter Olympic Games," IEEE Globecom 2016, Washington DC, USA, 4-8 Dec. 2016.

[5] 5G PAN-EUROPEAN TRIALS ROADMAP VERSION 2.0, available online in https://5g-ppp.eu

[6] The 5GENESIS project, available online: http://5genesis.eu/

[7] 5G-PPP Architecture Working Group, View on 5G Architecture, https://5g-ppp.eu/wpcontent/uploads/2018/01/5G-PPP-5G-Architecture-WhitePaper-Jan-2018-v2.0.pdf

[8] http://www.etsi.org/images/files/ETSIWhitePapers/etsi_wp24 _MEC_deployment_in_4G_5G_FINAL.pdf

[9] D. Tsolkas, et al, A survey on parametric QoE estimation for popular services, Journal of Network and Computer Applications, Volume 77, 2017, Pages 1-17, ISSN 1084-8045. 